\title{
Manejo anestésico para electroporación irreversible en el paciente pediátrico
}

\author{
Anesthetic management for irreversible \\ electroporation in the pediatric patient
}

\author{
Dra. Ivón Alejandra Moreno-Ravelo,* Dr. Plácido Sánchez-Acosta,* \\ Dra. Lina Andrea Sarmiento-Argüello*
}

Citar como: Moreno-Ravelo IA, Sánchez-Acosta P, Sarmiento-Argüello LA. Manejo anestésico para electroporación irreversible en el paciente pediátrico. Rev Mex Anestesiol. 2021; 44 (4): 277-281. https://dx.doi.org/10.35366/100873

RESUMEN. Introducción: La electroporación irreversible (EIR) es una técnica conocida desde 1972; fue incorporada al ámbito oncológico en el año 2005 como técnica de ablación tumoral basada en pulsos eléctricos cortos de alto voltaje y es utilizada para introducir elementos químicos por difusión, alterar la estructura genética celular, fusionar una célula con otra (reversible) o provocarle la muerte (irreversible). Estas descargas eléctricas conllevan un riesgo asociado de arritmias cardíacas, contracciones musculares severas y convulsiones. Mantener un adecuado plano anestésico, analgesia óptima, relajación neuromuscular profunda y electrocardiograma sincronizado a los pulsos eléctricos son los objetivos principales del manejo anestésico. Objetivo: Describir las implicaciones anestésicas de la EIR en el paciente pediátrico. Material y métodos: Revisión narrativa basada en una búsqueda de artículos relacionados con la EIR en las principales bases de datos, donde la principal fuente de información son los reportes de casos y las revisiones no sistemáticas de la literatura, tanto del área de anestesiología y dolor como de ingeniería, radiología intervencionista vascular y cardiovascular, y cirugía. Resultados: Se encontraron y revisaron un total de 17 referencias bibliográficas. Conclusión: La anestesia para EIR en el paciente pediátrico debe garantizar un adecuado plano anestésico idealmente con intubación endotraqueal, analgesia óptima, relajación profunda y control de arritmias cardíacas con sincronización del electrocardiograma.

ABSTRACT. Introduction: Irreversible electroporation (IRE) is a technique known since 1972 incorporated into the oncological field in 2005 as a tumor ablation technique based on short high-voltage electrical pulses used to introduce chemical elements by diffusion, alter the cellular genetic structure, fuse a cell with other (reversible) or cause death (irreversible). These electric shocks carry an associated risk of cardiac arrhythmias, severe muscle contractions and seizures. Maintain an adequate anesthetic plane, optimal analgesia, deep neuromuscular relaxation and an electrocardiogram synchronized to the electrical pulses are the main objectives of anesthetic management. Objective: Describe the anesthetic implications of IRE in the pediatric patient. Material and methods: Narrative review based on a search for articles related to IRE in the most important databases, where the main source of information is case reports and non-systematic reviews of the literature, both in the area of anesthesiology and pain as well as engineering, vascular and cardiovascular interventional radiology, and surgery. Results: A total of 17 bibliographic references were found and reviewed. Conclusion: The anesthesia for IRE in the pediatric patient should guarantee an adequate anesthetic plane ideally with endotracheal intubation, optimal analgesia, deep relaxation and control of cardiac arrhythmias with electrocardiogram synchronization.

\section{INTRODUCCIÓN}

L a electroporación irreversible (EIR) es una técnica conocida desde 1972 incorporada al ámbito oncológico en el 2005 como técnica de ablación tumoral basada en la apertura de un canal hidrofiílico en la membrana lipídica, utilizando impulsos eléctricos con ciclos de 90 pulsos, administrados generalmente en grupos de 8 a 10, de 70-100 microsegundos de duración y elevado voltaje (1,000-3.000 V), así como una intensidad de 20-50 A de corriente continua, el cual puede ser utilizado para introducir elementos químicos por difusión, alterar la estructura genética celular, fusionar una célula con otra

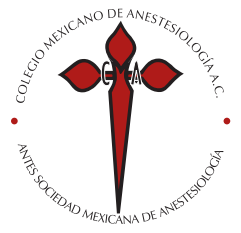

Palabras clave:

Técnicas de ablación, electroporación, anestesia general, arritmias cardíacas, relajación neuromuscular.

Keywords:

Ablation techniques, electroporation, general anesthesia, cardiac arrhythmias, muscle relaxation.

* Departamento de Anestesiología Pediátrica, Instituto Nacional de Pediatría.

Correspondencia:

Dra. Ivón Alejandra

Moreno-Ravelo

E-mail: vony2708@gmail.com

Recibido: 10-02-2020

Aceptado: 25-02-2021

Abreviaturas:

${ }^{\circ} \mathrm{C}=$ Grados Celsius.

$\mathrm{A}=$ Amperes.

ADN = Ácido desoxirribonucleico. ASA = Sociedad Americana de Anestesiología (American Society of Anesthesiologists). ATP = Adenosín trifosfato $\mathrm{BNM}=$ Bloqueo neuromuscular. $\mathrm{cm}=$ Centímetros $\mathrm{mm}=$ Milímetros. ECG $=$ Electrocardiograma . EIR = Electroporación irreversible. EVA = Escala visual análoga. $\mathrm{mmHg}=$ Milímetros de mercurio. TC = Tomografía computarizada. $\mathrm{U} / \mathrm{L}=$ Unidades/litro.

$\mathrm{V}=$ Voltios.

$\mathrm{V} / \mathrm{cm}=$ Voltios/centímetro. NYHA = Asociación del Corazón de Nueva York (New York Heart Association). $\mathrm{Hz}=$ Hercio. 
(reversible) o provocarle la muerte (irreversible) $)^{(1-5)}$. La particularidad de esta técnica es el hecho de no producir efectos secundarios como los térmicos, producidos por la ablación con radiofrecuencia, o cualquiera de los causados por la quimio o radioterapia ${ }^{(1-8)}$.

Sin embargo estas descargas eléctricas conllevan un riesgo asociado de arritmias cardiacas, contracciones musculares severas y convulsiones ${ }^{(4)}$. Mantener un adecuado plano anestésico, una analgesia óptima, una relajación neuromuscular profunda y un electrocardiograma sincronizado a los pulsos eléctricos son los objetivos principales del manejo anestésico $^{(4)}$. El objetivo de esta revisión es describir la técnica de EIR, sus ventajas, efectos adversos y contraindicaciones de la misma, y las implicaciones anestésicas en el paciente pediátrico.

\section{MATERIAL Y MÉTODOS}

Se realizó una búsqueda en las bases de datos PubMed, ClinicalKey, Google, con los términos MeSH: electroporación, electroporación irreversible, electroporación en niños, anestesia para electroporación, electroporación y anestesia. Se seleccionaron artículos en inglés y español, disponibles en texto completo, tanto del área de anestesiología y dolor como de ingeniería, radiología intervencionista vascular y cardiovascular, y cirugía. Se encontraron y revisaron un total de 17 referencias bibliográficas.

\section{DEFINICIÓN E HISTORIA DE LA EIR}

La electroporación irreversible (EIR), también conocida como electropermeabilización, es una nueva técnica de ablación de tumores basada en la aplicación local de un campo eléctrico entre dos o más electrodos insertados alrededor de un tumor. Esta técnica se comenzó a usar en procesos de esterilización de alimentos desde los años 60. La primera descripción fue por Nollet en 1754, quien aplicó chispas eléctricas a la piel de humano y de animal y notó puntos rojos resultantes ${ }^{(2)}$. En el siglo XVIII existe un informe del uso de descarga de alto voltaje para purificar el agua de río, y entre los años 1950 y 1960, se usó en experimentos de nervios y en estudios relacionados con la esterilización de $\operatorname{alimentos}^{(2)}$. A principios del año 1980, se introdujo para inducir fusión de células (electrofusión) y la transferencia de ácido desoxirribonucleico (ADN) dentro de las células (electrotransferencia), pero es a partir del año 2005 que se incorpora al ámbito oncológico como terapia ablativa alternativa a la cirugía. También tiene aplicaciones establecidas en electrotransferencia génica, terapia génica, fusión celular, electroquimioterapia, esterilización y procesamiento de alimentos ${ }^{(2-5)}$.

\section{DESCRIPCIÓN DE LA TÉCNICA}

La electroporación irreversible (EIR) se puede realizar de forma percutánea o abierta, esta última se realiza a través de una incisión superior en la línea media del cuerpo cuando se requiere tratar tumores que involucran la cavidad abdominal y/o pélvica. La adecuada colocación de la aguja es imprescindible, por lo que la confirmación se realiza por ultrasonido en caso de técnica abierta con mediciones que se ingresan en el software de la unidad de energía, lo que permite una tensión óptima y la entrega de la longitud del pulso. Se colocan dos o más electrodos de aguja de $15 \mathrm{~cm}$, con exposición de $2 \mathrm{~cm}$ (hígado, riñón y pelvis inferior) o 1.5 cm (páncreas). En los procedimientos percutáneos, los electrodos son avanzados bajo fluoroscopía por tomografía computarizada (TC) con o sin ultrasonido; para permitir la colocación de la aguja bajo fluoroscopía por TC, la ventilación del paciente se detiene brevemente ${ }^{(4,6,9)}$.

Las agujas se insertan paralelas entre sí; es importante evitar la convergencia o divergencia que puede resultar en una zona de ablación no uniforme. El espacio entre las sondas es crítico, el espacio ideal es entre 1.5 y $2 \mathrm{~cm}$ para un volumen de ablación más grande, la separación máxima de los electrodos es de $2.5 \mathrm{~cm}$. Espaciar más la distancia recomendada aumenta las posibilidades de errores de alta corriente. La zona de ablación se planifica para incluir el tumor y un margen de $1 \mathrm{~cm}^{(10-12)}$.

El efecto puede ser reversible o irreversible según la duración, el número de pulsos y la intensidad del campo eléctrico generado, esto produce cambios en la permeabilidad de la membrana, lo cual provoca la pérdida de la homeostasis, y finalmente, de acuerdo con los pulsos eléctricos aplicados, la muerte celular por necrosis ${ }^{(3)}$. A partir de modelos matemáticos se determinó que, una intensidad de campo mayor o igual a $100 \mathrm{~V} / \mathrm{cm}$ (umbral para la electroporación reversible) se extiende a un radio de $1.7 \mathrm{~cm}$ desde cada electrodo unipolar y una intensidad de campo mayor a $500 \mathrm{~V} / \mathrm{cm}$ (umbral para la electroporación irreversible) para electrodos a $2 \mathrm{~cm}$, formándose una lesión elipsoidal de $30 \times 25 \times 17$ mm aproximadamente ${ }^{(13)}$.

Los múltiples ciclos de pulsos eléctricos cortos y de muy alto voltaje alteran el potencial transmembrana de las células tumorales, lo que lleva a la creación de defectos a nanoescala en la bicapa lipídica de la membrana celular, lo cual aumenta la permeabilidad y provoca tensión osmótica, química y liberación de calcio intracelular desde el retículo endoplásmico. Los impulsos eléctricos también pueden incrementar la permeabilidad mitocondrial, liberando citocromo $\mathrm{C}$, la cual es una proteína pequeña involucrada en la iniciación de la apoptosis.

Por otra parte, puede haber daño al ADN y niveles elevados de radicales libres de oxígeno, induciendo apoptosis mediada por tensión oxidante. El voltaje estándar predeterminado es 
de 1,500 voltios por centímetro, se inicia con un suministro planificado de 90 pulsos y un ancho de pulso de 70 a 90 microsegundos y 20-50 A. De manera inicial, se administran 20 pulsos y luego se detiene la entrega para evaluar el amperaje y establecer el voltaje y la amplitud de pulso óptimos. La permeabilidad de la membrana se hace permanente y la célula finalmente muere debido a la pérdida de homeostasis, se destruye efectivamente todas las células dentro del área de ablación y la matriz extracelular se conserva ${ }^{(2,4,9)}$.

La célula intenta restaurar los gradientes de iones y el potencial de membrana activando las bombas de iones y reparando la membrana. El alto gasto de energía para estos procesos puede verse agravado por la fuga de adenosín trifosfato (ATP) fuera de la célula a través de los electroporos. Se cree que una alta demanda prolongada de ATP combinada con la pérdida de ATP durante exposiciones más largas podría ser un factor responsable de la sensibilización. Otro mecanismo potencial de electrosensibilización puede implicar daño oxidativo directo o indirecto a la membrana por la exposición, lo que aumentaría su susceptibilidad a la permeabilización ${ }^{(5)}$.

\section{VENTAJAS DE LA EIR}

Es una técnica para el manejo de muchos tipos de tumores de tejidos blandos localmente avanzados que han tenido mala respuesta a quimioterapia, radioterapia o recurrencia, e incluyen tumores hepáticos no resecables perivasculares, colangiocarcinoma perihiliar no resecable y colangiocarcinoma intrahepático, cáncer pancreático localmente avanzado, tumores retroperitoneales perivasculares y rabdomiosarco$\mathrm{ma}^{(7-9,14)}$.

Presenta dos principales ventajas respecto a otras técnicas de ablación tumoral: no afecta de forma significativa al tejido circundante como vasos, nervios y conductos biliares y no alcanza los $50^{\circ} \mathrm{C}$; esta temperatura constituye el umbral del denominado efecto heat-sink, en el que existe una pérdida de calor a través del flujo sanguíneo cerca de grandes vasos y que puede disminuir la efectividad de la ablación o presentar ablación incompleta, como se observa en otros métodos como la crioablación, radiofrecuencia o ablación de microondas, los cuales involucran enfriamiento o calentamiento en tejido para inducir la muerte celular y llevan cierto riesgo al entorno extracelular adyacente ${ }^{(2-4,8)}$.

La EIR tiene una ventaja sobre la ablación térmica en tumores peribiliares localizados a menos de $1 \mathrm{~cm}$ de los conductos biliares principales, ya que se observa menor riesgo de lesión colateral y destrucción completa y reducción marcada del tumor en dos semanas ${ }^{(10)}$.

Además, existe evidencia de modelos experimentales que apoyan el uso de electroporación cerca de las semillas de braquiterapia u otros implantes metálicos pequeños, sin alterar significativamente el comportamiento del pulso eléctrico o las dimensiones de la lesión ${ }^{(15)}$.

\section{CONTRAINDICACIONES DE LA EIR}

Las contraindicaciones son: pacientes en tratamiento con antiarrítmicos o con marcapasos o desfibrilador automático implantable; antecedentes de insuficiencia cardíaca congestiva (clase NYHA superior a II); enfermedad activa de las arterias coronarias; hipertensión no controlada. En caso de ser necesario, se recomienda colocar parches de desfibrilación externos conectados como medida de precaución ${ }^{(4)}$. Una contraindicación relativa son crisis convulsivas recientes o no controladas y/o epilepsia ya diagnosticada.

\section{RETOS ANESTÉSICOS EN LA EIR}

La aplicación de pulsos eléctricos de alto voltaje puede ocasionar complicaciones diversas y presentar desafíos anestésicos específicos.

La ubicación para la realización de este procedimiento suele ser «fuera de quirófano» por precisar técnicas de imagen complejas (sala de TC o de intervencionismo), lo que dificulta la monitorización completa del paciente dada la accesibilidad limitada y la infraestructura incorrecta, ya que la necesidad de anestesia no es planeada durante el diseño de estos lugares. Éstos deben cumplir con los estándares para «anestesia fuera de quirófano» recomendados por la Sociedad Americana de Anestesiología (ASA). Todos los procedimientos para electroporación requieren anestesia general y la monitorización es un aspecto fundamental ${ }^{(3,4)}$.

Los cambios en la permeabilidad de las membranas celulares modifican el transporte iónico, lo que puede generar arritmias (extrasístoles ventriculares, taquicardia ventricular y fibrilación ventricular), por lo cual una doble monitorización electrocardiográfica sería lo ideal, una para sincronizar los pulsos de la electroporación con el electrocardiograma (ECG) y otra para el control electrocardiográfico per se. Deben ser estables y libres de artefactos para permitir una correcta sincronización. Los estímulos eléctricos externos administrados durante el período refractario absoluto del corazón, es decir, tras la onda R, son incapaces de inducir un potencial de acción. Existe un dispositivo de sincronización cardíaca preciso de tal modo que si la señal del ECG no es adecuada y consistente, la energía no se suministra ${ }^{(3,4,9)}$. La literatura reporta presencia de arritmias cardíacas menores transitorias sin consecuencias hemodinámicas y se ha considerado la fibrilación auricular preexistente como una contraindicación, ya que la taquicardia puede alterar la liberación de los pulsos ${ }^{(3,4,9)}$. Cuando el pulso se aplica a menos de $3 \mathrm{~cm}$ de distancia del corazón interfiere directamente con su sistema de conducción eléctrica, y a menos de $1.7 \mathrm{~cm}$ del corazón ocurren eventos fatales ${ }^{(3,13)}$. 
También es obligatoria la monitorización de la presión arterial. Se presenta hipertensión rápida tras la aplicación de los pulsos eléctricos, incluso con un manejo anestésico profundo y un buen bloqueo neuromuscular, esta hipertensión por lo general es transitoria. En adultos con presión sistólica mayor a $140 \mathrm{mmHg}$ y diastólica mayor a $88 \mathrm{mmHg}$ antes del inicio del suministro de energía produjo una hipertensión importante con presión sistólica mayor a 180 y diastólica mayor a 100 . Por lo general, la presión arterial sistólica aumenta a $44 \mathrm{mmHg}$ (rango de 27 a $108 \mathrm{mmHg}$ ) y la diastólica a $19 \mathrm{mmHg}$ (rango de 1 a $50 \mathrm{mmHg}$ ) en el adulto, no se cuentan con valores pediátricos. El mecanismo exacto detrás de este aumento no está claro, pero la estimulación del sistema nervioso autónomo es una explicación probable $e^{(3,4,16)}$.

Es importante tener en cuenta que la localización de la aguja en el área del tronco celíaco ha mostrado una asociación con eventos cardíacos. Esto es consistente con los hallazgos de Nielsen que describe que las mayores desviaciones en los parámetros de frecuencia cardíaca y presión arterial son en pacientes que se someten a electroporación en el área del páncreas secundario a la estimulación del plexo celíaco, se sabe que tiene un impacto en la hemodinámica por sus fibras simpáticas ${ }^{(14)}$.

Otro aspecto a considerar es la monitorización del bloqueo neuromuscular (BNM), ya que se requiere un nivel profundo de BNM para administrar de forma segura las descargas y minimizar cualquier trauma con las agujas. Un inadecuado BNM crea movimiento en el órgano diana, debido a que el músculo circundante se contrae y las agujas no están ancladas. Sin embargo, se ha reportado que las contracciones locales son más profundas durante los procedimientos percutáneos, especialmente cuando se insertaron electrodos a través de los músculos grandes; la corriente induce un campo electromagnético regional que causa la despolarización muscular directa, que no se evita del todo mediante el uso de relajantes neuromusculares, incluso se reportan contracciones diafragmáticas, aun con buena relajación $n^{(2,4,8,9,16)}$. Se pueden observar contracciones de la parte superior del cuerpo en pacientes paralizados inadecuadamente, incluso si se asegura una relajación muscular completa $^{(17)}$. Se sugiere el uso de rocuronio por la facilidad de reversión de su efecto con el uso de sugammadex en procedimientos de corta duración.

La EIR puede desencadenar actividad epileptógena cerebral cuyo mecanismo aún no está dilucidado. Sin embargo, los pulsos eléctricos no parecen causar actividad cerebral reactiva que alcancen la frecuencia mínima de $5 \mathrm{~Hz}$ requerida para provocar una convulsión. Durante la terapia electroconvulsiva (en el caso de la depresión resistente a la terapia farmacológica) las crisis son inducidas por la aplicación de pulsos eléctricos de 5 Hz o más al cerebro, así el riesgo de convulsiones durante la electroporación es probablemente muy bajo. De ser necesario el uso de EIR en pacientes con epilepsia sugerimos la vigilan- cia cerebral durante el procedimiento para un reconocimiento rápido de las crisis y el inicio del tratamiento oportuno ${ }^{(3,4,17)}$.

\section{RECOMENDACIONES PARA EL MANEJO ANESTÉSICO EN EIR}

La mayoría de los procedimientos duran aproximadamente 200 minutos y el anestésico inhalado utilizado para el mantenimiento usualmente es el sevoflurano. Todos los procedimientos para electroporación requieren anestesia general e intubación orotraqueal debido al riesgo de laringoespasmo y/o broncoespasmo en caso de que el paciente se encuentre en un inadecuado plano anestésico. Se requiere monitoreo con ECG doble para la adecuada sincronización cardíaca, presión arterial no invasiva y monitorización del BNM con bloqueo profundo garantizando un tren de cuatro de 0/4. Se han reportado alternativas en el manejo anestésico con el uso de remifentanilo en infusión continua con bloqueo epidural $^{(4,6,9)}$.

Respecto al dolor, éste suele ser leve en las primeras 24 horas después de la electroporación percutánea con una puntuación de tres en la escala visual análoga (EVA). En la mayoría de los pacientes el dolor posterior al procedimiento puede tratarse con analgésicos orales. El puntaje de EVA más alto reportado ha sido después del tratamiento pancreático, con mayor dolor en las primeras 24 horas y puntuaciones máximas altas de nueve, que pueden explicarse por la ubicación anatómica del páncreas cerca del plexo celíaco, combinado con la inducción de una pancreatitis reactiva causada por el tratamiento $^{(4,17)}$.

En el postoperatorio se deben monitorizar los electrolitos séricos (en especial el potasio por el riesgo de hipercalemia), la función renal, las enzimas pancreáticas y hepáticas; estas últimas presentan un aumento, alcanzando el nivel más alto el primer día después del procedimiento ${ }^{(4,14,17)}$. En los procedimientos de tumores hepáticos se ha reportado un aumento transitorio esperado a las 24 horas de la enzima hepática alanina aminotransferasa (un marcador de daño celular agudo) con aumentos de 19-1,747 U/L, volviendo a niveles normales o iniciales al mes después del procedimiento ${ }^{(11)}$.

\section{LIMITACIONES}

El objetivo de este artículo es la revisión de las implicaciones anestésicas de la EIR en pacientes pediátricos. Sin embargo, la calidad de la evidencia es baja (reportes de caso y revisiones de tema) y la información se presenta de manera descriptiva. Existe la necesidad de crear protocolos anestésicos óptimos para garantizar una terapia EIR segura, efectiva y exitosa. No obstante, se requieren estudios con mejor calidad metodológica; dada la baja frecuencia con la que se realiza este procedimiento, así como la falta de equipos adecuados para su 
implementación, probablemente sea difícil encontrar artículos con mayor nivel de evidencia.

\section{CONCLUSIONES}

La anestesia para EIR en el paciente pediátrico debe garantizar un adecuado plano anestésico idealmente con intubación endotraqueal, una analgesia óptima, un BNM profundo, un adecuado control de la hipertensión (en el caso de pacientes con zonas de ablación grandes o en tumores pancreáticos) y control de arritmias cardíacas con sincronización del ECG. La anestesia general combinada con bloqueo neuroaxial (caudal, epidural o espinal) puede ser útil en estos pacientes.

\section{Conflicto de intereses: Ninguno.}

\section{REFERENCIAS}

1. Villaverde J. Instrumentos para electroporación reversible e irreversible, con simulaciones de electroporación con el programa Quick-Field. Uruguay: Núcleo de Ingeniería Biomédica Universidad de la Republica; 2011. pp. 1-7.

2. Deipolyi AR, Golberg A, Yarmush ML, Arellano RS, Oklu R. Electroporación irreversible: evolución de una técnica de laboratorio en oncología de intervención. Radiología de Intervención de Diagnóstico. 2014;20:147-154.

3. Flor-Robledo M, Solís-Muñoz P, Sanjuán-Álvarez M, AbadalVillayandre JM, Asensio-Merino F. Electroporación percutánea irreversible de un tumor renal: manejo anestésico. Rev Esp Anestesiol Reanim. 2016;63:419-422. http://dx.doi.org/10.1016/j.redar.2015.11.011

4. Nielsen K, Scheffer HJ, Vieveen JM, van Tilborg AA, Meijer S, van Kuijk C, et al. Anaesthetic management during open and percutaneous irreversible electroporation. Br J Anaesth. 2014;113:985-992. doi: $10.1093 / \mathrm{bja} / \mathrm{aeu} 256$

5. Pakhomova ON, Gregory BW, Khorokhorina VA, Bowman AM, Xiao S, Pakhomov AG. Electroporation-induced electrosensitization. PLoS One. 2011;6:e17100. doi: 10.1371/journal.pone.0017100.

6. Ball C, Thomson KR, Kavnoudias H. Irreversible electroporation: a new challenge in "out of operating theater" anesthesia. Anesth Analg. 2010;110:1305-1309. doi: 10.1213/ANE.0b013e3181d27b30.

7. Heller R, Davalos RV. Special collection on electroporation-based therapies: a selection of papers from the second world congress on electroporation. Technol Cancer Res Treat. 2019;18:1533033819852966. doi: $10.1177 / 1533033819852966$

8. Thomson KR, Kavnoudias H, Neal RE 2nd. Introduction to irreversible electroporation--principles and techniques. Tech Vasc Interv Radiol. 2015;18(3):128-1234. doi: 10.1053/j.tvir.2015.06.002.

9. Martin RC, Schwartz E, Adams J, Farah I, Derhake BM. Intra-operative anesthesia management in patients undergoing surgical irreversible electroporation of the pancreas, liver, kidney, and retroperitoneal tumors. Anesth Pain Med. 2015;5:e22786. doi: 10.5812/aapm.22786.

10. Narayanan G. Irreversible electroporation. Semin Intervent Radiol. 2015;32:349-355. doi: 10.1055/s-0035-1564706.

11. Thomson KR, Cheung W, Ellis SJ, Federman D, Kavnoudias H, LoaderOliver D, et al. Investigation of the safety of irreversible electroporation in humans. J Vasc Interv Radiol. 2011;22:611-621. doi: 10.1016/j. jvir.2010.12.014.

12. Li W, Fan Q, Ji Z, Qiu X, Li Z. The effects of irreversible electroporation (IRE) on nerves. PLoS One. 2011;6:e18831. doi: 10.1371/journal. pone.0018831.

13. Deodhar A, Dickfeld T, Single GW, Hamilton WC Jr, Thornton $\mathrm{RH}$, Sofocleous CT, et al. Irreversible electroporation near the heart: ventricular arrhythmias can be prevented with ECG synchronization. AJR Am J Roentgenol. 2011;196:W330-W335. doi: 10.2214/ AJR.10.4490.

14. Kambakamba P, Bonvini JM, Glenck M, Castrezana López L, Pfammatter T, Clavien PA, et al. Intraoperative adverse events during irreversible electroporation-a call for caution. Am J Surg. 2016;212:715721. doi: 10.1016/j.amjsurg.2016.07.001.

15. Neal RE 2nd, Smith RL, Kavnoudias H, Rosenfeldt F, Ou R, Mclean $\mathrm{CA}$, et al. The effects of metallic implants on electroporation therapies: feasibility of irreversible electroporation for brachytherapy salvage. Cardiovasc Intervent Radiol. 2013;36:1638-1645. doi: 10.1007/s00270013-0704-1.

16. Satava R. Electroporation. Anesth Analg. 2010;110:1264. doi: 10.1213/ ANE.0b013e3181d8cb74.

17. Vieveen JM, Bouwman RA. Anesthetic management during irreversible electroporation procedures. In: Meijerink M, Scheffer H, Narayanan G (eds.). Irreversible electroporation in clinical practice. Springer, Cham. 2018. https://doi.org/10.1007/978-3-319-55113-5_6 